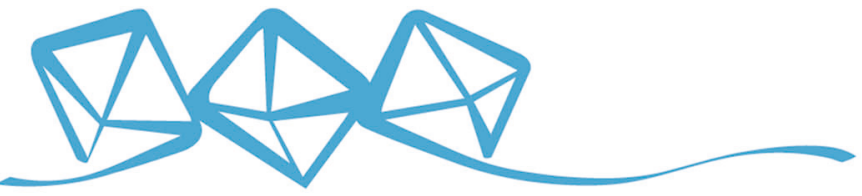 COMMUNICATIONS MATERIALS
}

ARTICLE

https://doi.org/10.1038/s43246-020-00065-3 OPEN

\section{Water droplet friction and rolling dynamics on superhydrophobic surfaces}

\author{
Matilda Backholm (10 ${ }^{1 凶}$, Daniel Molpeceres ${ }^{1}$, Maja Vuckovac (10 1, Heikki Nurmi (1) ${ }^{1}$, Matti J. Hokkanen ${ }^{1,2}$, \\ Ville Jokinen (1D ${ }^{3}$, Jaakko V. I. Timonen (1) ${ }^{1}$ \& Robin H. A. Ras (i] ${ }^{1,4 凶}$
}

Superhydrophobicity is a remarkable surface property found in nature and mimicked in many engineering applications, including anti-wetting, anti-fogging, and anti-fouling coatings. As synthetic superhydrophobic coatings approach the extreme non-wetting limit, quantification of their slipperiness becomes increasingly challenging: although contact angle goniometry remains widely used as the gold standard method, it has proven insufficient. Here, micropipette force sensors are used to directly measure the friction force of water droplets moving on super-slippery superhydrophobic surfaces that cannot be quantified with contact angle goniometry. Superhydrophobic etched silicon surfaces with tunable slipperiness are investigated as model samples. Micropipette force sensors render up to three orders of magnitude better force sensitivity than using the indirect contact angle goniometry approach. We directly measure a friction force as low as $7 \pm 4 \mathrm{nN}$ for a millimetric water droplet moving on the most slippery surface. Finally, we combine micropipette force sensors with particle image velocimetry and reveal purely rolling water droplets on superhydrophobic surfaces.

\footnotetext{
${ }^{1}$ Department of Applied Physics, Aalto University, P.O. Box 15100, 02150 Espoo, Finland. ${ }^{2}$ Department of Electrical Engineering and Automation, Aalto University, P.O. Box 15500, 01250 Espoo, Finland. ${ }^{3}$ Department of Chemistry and Materials Science, Aalto University, P.O. Box 16100, 02150 Espoo, Finland.

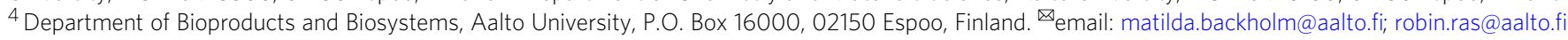


W hile friction is essential in everyday activities, such as walking and driving, it is also responsible for $\sim 23 \%$ of the world's total energy consumption ${ }^{1}$. By introducing new friction-reducing coatings in the fields of transportation and power generation alone, notable economic and environmental savings could be achieved, including a reduction in global $\mathrm{CO}_{2}$ emissions. One example is a durable superhydrophobic coating ${ }^{2}$, which could greatly enhance the long-term efficiency of solar cells (via self-cleaning surface finish) or reduce the costs of transporting goods on ships ${ }^{2}$.

In order to produce coatings of increasingly high quality, the manufacturing process has to be guided by a sensitive characterization tool of the non-wetting properties ${ }^{3}$. Today, contact angle goniometry (CAG) is the conventional experimental tool for probing water-repellency on various surfaces ${ }^{4}$. In this technique, the profile of a growing and shrinking or moving droplet is observed optically and the resulting advancing and receding contact angles $\left(\theta_{\mathrm{a}}\right.$ and $\theta_{\mathrm{r}}$, Fig. 1a) are determined through subsequent image analysis. The friction force (also referred to as the lateral adhesion force) is then calculated (assuming a circular contact region) as ${ }^{5-7}$

$$
F_{\mathrm{LA}}=\frac{24}{\pi^{3}} \gamma D\left(\cos \theta_{\mathrm{r}}-\cos \theta_{\mathrm{a}}\right)
$$

where $D$ is the diameter of the contact region and $\gamma$ is the surface tension ( $\gamma=0.0728 \mathrm{~N} \mathrm{~m}^{-1}$ for water) (Fig. 1b). The challenge is that when surfaces become increasingly slippery, the advancing and receding contact angles approach each other (decreasing contact angle hysteresis, $\theta_{\mathrm{a}}-\theta_{\mathrm{r}}$ ) and quantification of slipperiness by contact angle will be limited by accuracy of image analysis. This is true for various slippery surfaces, such as lubricantinfused 8,9 and slippery omniphobic covalently attached liquid (SOCAL) $\operatorname{surfaces}^{9}\left(\theta \approx 90^{\circ}\right)$, as well as liquid-like silicone brush surfaces $^{10}\left(\theta \approx 20-100^{\circ}\right)$. Further issues arise on superhydrophobic surfaces (defined by high contact angles $\theta \gtrsim 150^{\circ}$ and low contact angle hysteresis $\left.\theta_{\mathrm{a}}-\theta_{\mathrm{r}} \lesssim 10^{\circ}\right)^{11}$ where optical distortions close to the contact region render increasingly high errors $\left(\theta_{\text {err }} \approx \pm(1-10)^{\circ}\right.$ for $\left.\theta=(150-180)^{\circ}\right)$ in the contact angle analysis ${ }^{12-14}$. For next-generation slippery surfaces, Eq. (1) suggests that reducing the contact angle hysteresis could lead to significant reduction in the forces holding the droplet on the surface (Fig. 1b). This can, however, only be assessed by measuring the forces directly.
The tilt-stage $4,5,15,16$ and oscillating droplet tribometer ${ }^{17}$ techniques have been developed to measure droplet friction forces. One further, especially important emerging technique is the use of force-calibrated elastic cantilevers (spring constant $k_{\mathrm{p}}$ ) to measure the friction force of droplets moving on various substrates ${ }^{7-9,18-20}$, including moderately superhydrophobic ${ }^{7,9,19}$, SOCAL $^{9,20}$, and lubricated ${ }^{8,9}$ coatings. The force is directly obtained through optical detection of the deflection $(\Delta x)$ of the cantilever: $F=-k_{\mathrm{p}} \Delta x$. Various kinds of cantilevers have been used to study liquid-repellent surfaces, including thick rectangular glass capillaries (side lengths $0.04-0.4 \mathrm{~mm}, k_{\mathrm{p}}=100-200 \mathrm{nN} \mu \mathrm{m}^{-1}$, force resolution $\sim 40 \mathrm{nN})^{7,19}$ and thick polymeric tubes (inner/

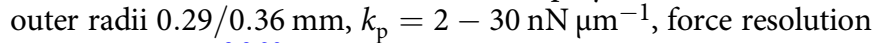
$\sim 10-100 \mathrm{nN})^{8,9,20}$. The main downside of those cantilevers is that their width is comparable to the droplet size, which affects the droplet shape and disturbs the flow inside the droplet. This prevents an accurate study of the droplet fluid dynamics-a major open question still being whether droplets roll or slide on superhydrophobic surfaces ${ }^{21}$. Whereas high-viscosity droplets are known to roll down superhydrophobic surfaces ${ }^{22,23}$, direct particle image velocimetry (PIV) experiments have reported the motion of water droplets to transition from roll-slip motion on hydrophobic surfaces $^{24,25}$ to pure sliding on superhydrophobic surfaces ${ }^{26,27}$. This slip motion is common for water moving on superhydrophobic surfaces ${ }^{28}$, where the liquid-air interface (between the no-slip, liquid-solid contact points) can be assumed to be shear-free $^{29}$. Previous PIV studies have been performed on tilted planes with very high droplet velocities. A systematic, velocitycontrolled study of the internal droplet flow dynamics is, however, still lacking on superhydrophobic surfaces.

In this work, we introduce micropipette force sensors (MFS) as what can be considered as ideal cantilevers for droplet friction measurements. The deflection of a long $(\sim \mathrm{cm})$ and thin $(\sim \mu \mathrm{m})$ hollow glass cantilever is used to measure friction forces as low as a few nN. Five different superhydrophobic etched silicon substrates are studied as model samples with varying slipperiness (contact angle hysteresis). We show that MFS is superior to CAG in quantifying the friction force on all samples. Due to the high sensitivity of MFS, it can also be used on super-slippery (contact angle hysteresis approaching zero) superhydrophobic samples that have been previously inaccessible by other techniques. Finally, we combine MFS with PIV to simultaneously measure both the friction force and the internal fluid dynamics of the
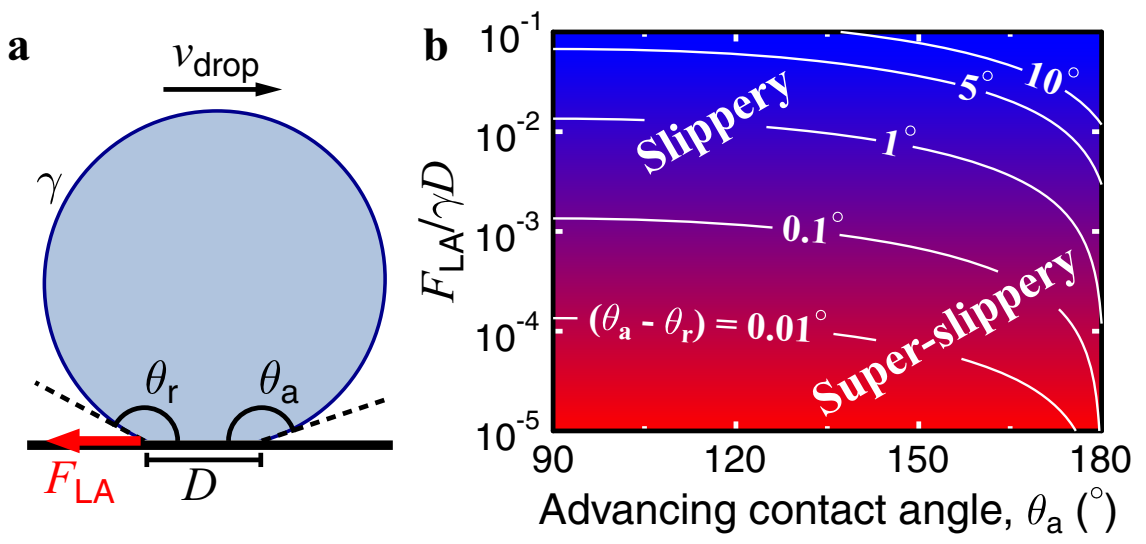

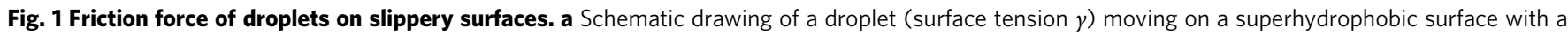
constant speed $v_{\mathrm{drop}}$. The motion is opposed by the friction force $F_{\mathrm{LA}}$, which is a function of the contact region diameter ( $D$ ) and the advancing and receding contact angles $\left(\theta_{a}=166^{\circ}\right.$ and $\theta_{r}=156^{\circ}$ in this example). $\mathbf{b}$ The dimensionless theoretical friction force calculated using Eq. (1) plotted as a function of advancing contact angle for different contact angle hysteresis $\left(\theta_{a}-\theta_{r}\right)$ values. On super-slippery surfaces, the contact angle hysteresis decreases to unmeasurably low values around $F_{\mathrm{LA}} / \gamma D \lesssim 10^{-3}$ 

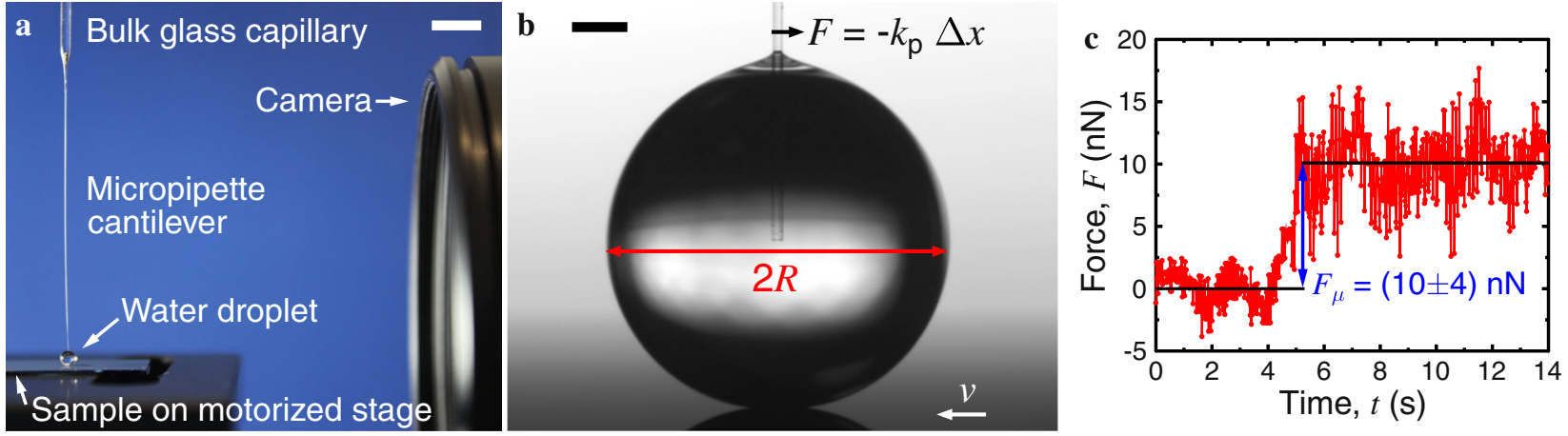

Fig. 2 Micropipette force sensor measurements of the friction of water droplets moving on superhydrophobic surfaces. a Photograph of the MFS setup with a millimetric water droplet on a superhydrophobic surface. The droplet is attached through capillary forces to a force-calibrated micropipette cantilever (pulled from a $1 \mathrm{~mm}$ thick glass capillary). The experiment is recorded with a camera from the side. Scale bar 5 mm. $\mathbf{b}$ Photograph of water droplet (radius $R=620 \pm 8 \mu \mathrm{m}$, contact region diameter $D=210 \pm 20 \mu \mathrm{m}$ ) on the spikes surface (sample A). During the experiment (see Supplementary Movie 1), the droplet is initially pulled along the substrate (resting on a motorized $x y z$-translational stage that starts moving to the left at time $\sim 5 \mathrm{~s}$ with a constant speed of $\left.v=0.1 \mathrm{~mm} \mathrm{~s}^{-1}\right)$ until the elastic force ( $F=-k_{\mathrm{p}} \Delta x$, where $k_{\mathrm{p}}$ is the spring constant of the pipette obtained through calibration) from the deflected $(\Delta x)$ micropipette matches the kinetic friction force $\left(F_{\mu}\right)$ of the substrate. At this point, the micropipette deflection remains nearly constant while the droplet slides along the surface. Scale bar $200 \mu \mathrm{m}$. c Force as a function of time from a typical experiment (same as in b). The average equilibrium (zero-force) position of the micropipette was first recorded for $\sim 5$ after which the surface started moving. The difference between the average zero and kinetic plateau force gives the kinetic friction force $\left(F_{\mu}\right)$. The error includes the standard deviation of both averages.

water droplet. Our experiments reveal the first observation of pure rolling motion of slowly moving water droplets on a superhydrophobic surface, and explore the transition to roll-slip motion as the droplet speed is increased.

\section{Results and discussion}

Micropipette force sensor measurements. Micropipette force sensors have recently been extensively used in various biophysi$\mathrm{cal}^{30-39}$ and soft matter ${ }^{40,41}$ studies (see ref. ${ }^{42}$ for a complete review and protocol and ref. ${ }^{43}$ for original paper) and consist of a macroscopically thick and robust millimetric, holdable glass capillary and a much thinner microscopic cantilever tip (Fig. 2a and Supplementary Fig. 1). The force sensors are easy to prepare using well-established fabrication protocols from inexpensive glass capillaries $^{42}$ (Methods). The high elastic modulus of glass enables simultaneously high spring constant $k_{\mathrm{p}}$ with extremely small diameter of the cantilever. Here, we demonstrate this using an $1.9-2.5 \mathrm{~cm}$ long MFS with inner/outer radii $\sim 15 / 20 \mu \mathrm{m}$,

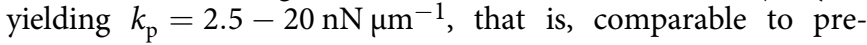
viously used cantilevers but an order of magnitude smaller diameter. A typical MFS friction experiment is shown in Fig. 2b (Supplementary Movie 1), rendering force versus time data (Fig. 2c) from which the experimental kinetic friction force $\left(F_{\mu}\right)$ is determined. The MFSs induce only miniscule deformation to droplets (Fig. 2b and Supplementary Fig. 2), yet achieve a force resolution as low as $\sim 4 \mathrm{nN}$ (Fig. 2c, see Supplementary Note 1), which is $\sim 2-25$ times better than in previous cantileverbased studies. In addition to having exceptional mechanical characteristics, MFS allows for convenient dispensing of the probe liquid, which is also utilized in the calibration of the pipette (Methods).

Friction on superhydrophobic etched silicon substrates. The performance of the MFS technique is demonstrated by measuring friction forces on a set of five different solid superhydrophobic surfaces ranging from slippery to super-slippery. These were prepared by maskless cryogenic deep reactive ion etching of silicon substrates to create a micro/nanostructure, followed by a subsequent coating with fluoropolymer through plasma-enhanced chemical vapor deposition (Methods) ${ }^{44}$. Slipperiness was controlled in the etching step by varying the ratio between $\mathrm{SF}_{6}$ and $\mathrm{O}_{2}$ gas flow rates, resulting in different surface topographies ranging gradually from spikes (sample A) to grass (sample E) (Fig. 3a-e, Supplementary Figs. 3-5). To compare our direct MFS force measurements with the existing gold standard method, CAG was used to measure the contact angles of the surfaces (Supplementary Fig. 6 and Supplementary Table 1, Methods).

As predicted by Eq. (1), the friction force increases linearly with increasing apparent contact region diameter (Fig. 3f). It is noteworthy that a millimetric water droplet (weight $\sim 10 \mu \mathrm{N}$ ) moving on the most slippery spikes surface (sample A) experiences the remarkably low friction force of only $7 \pm 4 \mathrm{nN}$ (Supplementary Table 2). The experimental force data are in excellent quantitative agreement with Eq. (1) (Fig. 3g). CAG proves to be too inaccurate to reliably distinguish between the five types of superhydrophobic samples (Supplementary Table 2). However, the differences between all samples can clearly be probed with MFS (Fig. 3f), including distinguishing between the

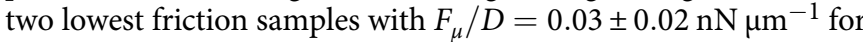

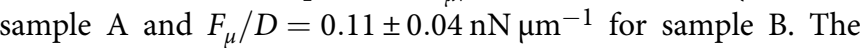
theoretical $F_{\mathrm{LA}}$ relies on the optically determined advancing and receding contact angles from each individual MFS experiment. The resulting relative friction force error $(\delta F=\Delta F / F$, where $\Delta F$ is the absolute error, see Supplementary Note 2 for error analysis) is more than ten times higher for the calculated force $\left(\delta F_{\mathrm{CAG}}\right)$ than for the force measured with MFS ( $\left.\delta F_{\mathrm{MFS}}\right)$ (Fig. $\left.3 \mathrm{~h}\right)$. As the samples become increasingly slippery (decreasing $F_{\mu} / D$ ), the direct MFS measurements are as much as three orders of magnitude more precise than using Eq. (1). For smaller droplets on the most slippery spikes sample, the lower limit of MFS is approached with decreasing signal-to-noise ratios and increasing relative force errors (see Supplementary Note 3 and Fig. 7). Our direct force measurements on this sample are, however, still ten times more precise than using Eq. (1), highlighting the exceptional suitability of MFS on such a super-slippery sample.

The friction of our etched silicon samples spans a very wide force range due to their topographical differences, where the most sparse spikes of sample A allow for an ultralow dimensionless friction force $F_{\mu} / \gamma D=(4 \pm 3) \cdot 10^{-4}$ that overcomes the friction of previously studied superhydrophobic surfaces made of micropillars ${ }^{9,45}$, SOCAL ${ }^{9}$, and silicone nanofilaments ${ }^{19}$, as well as lubricated surfaces ${ }^{9}$ and a "nearly friction- and adhesion-free" 

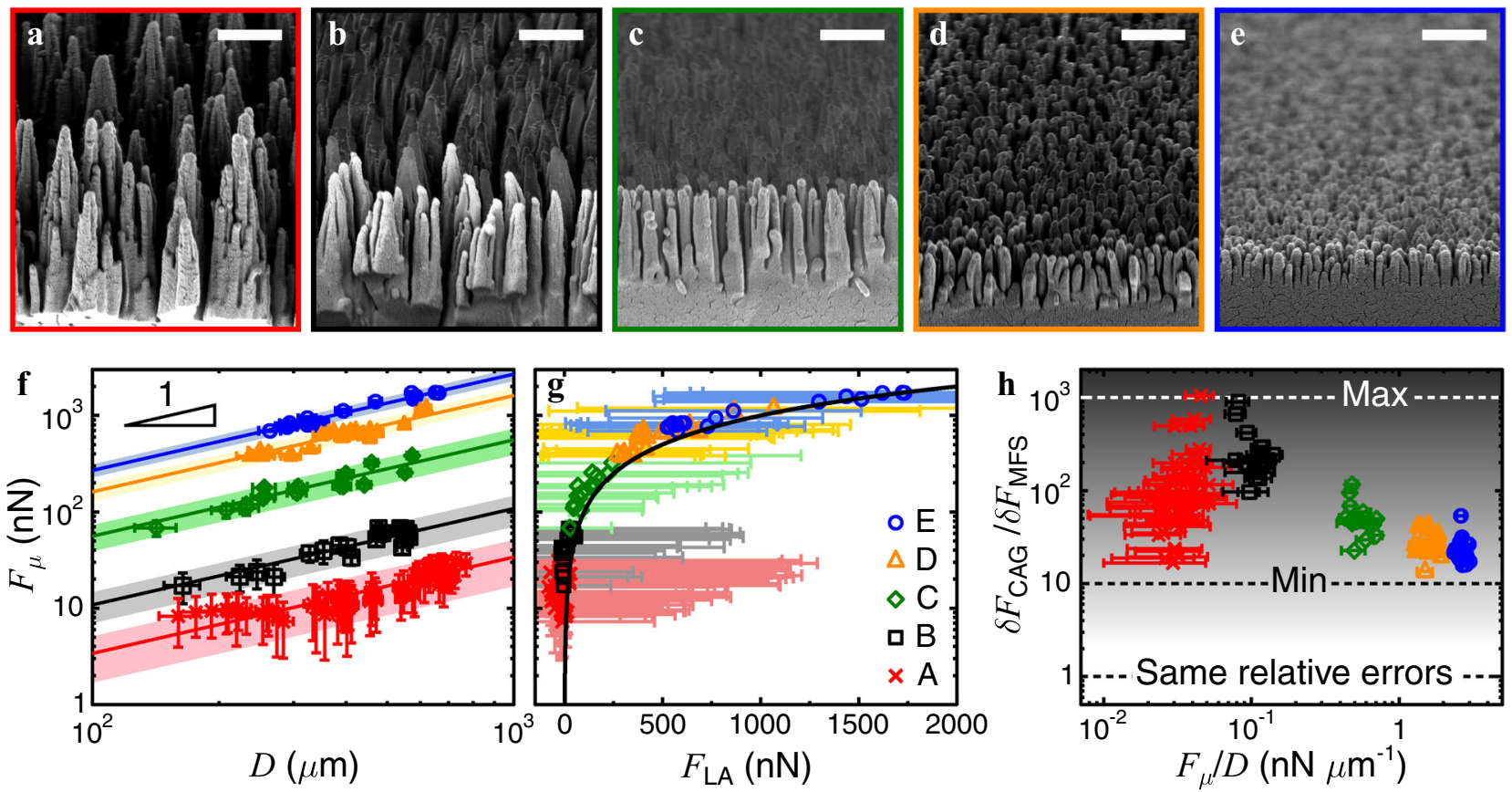

Fig. 3 Friction force on superhydrophobic etched silicon substrates-experiment vs. theory. a-e Scanning electron microscopy images taken at a 45 angle of the A (spikes), B, C, D, and E (grass) samples, respectively. Scale bars $1 \mu \mathrm{m}$. $\mathbf{f}$ Measured kinetic friction force as a function of contact region diameter. The solid lines are linear fits (through origin) to the data with the slopes $F_{\mu} / D=(2.7 \pm 0.4),(1.6 \pm 0.3),(0.6 \pm 0.2),(0.11 \pm 0.04),(0.03 \pm 0.02)$ $\mathrm{nN} \mu \mathrm{m}^{-1}$ for the $\mathrm{E}$ (grass), D, C, B, and A (spikes) samples, respectively. $\mathbf{g}$ Measured friction force as a function of the theoretically calculated lateral adhesion force $\left(F_{\mathrm{LA}}\right.$, Eq. (1)). The solid line (going though origin) has a slope of unity. $\mathbf{h}$ The ratio between the relative errors $(\delta F=\Delta F / F$, where $\Delta F$ is the absolute error) of the theoretical (CAG) and experimental (MFS) force estimates as a function of $F_{\mu} / D$. Measuring the friction force with MFS renders from $10(\mathrm{~min})$ to $1000(\mathrm{max})$ times more precise results as the surface becomes more slippery. As the lower limit of the micropipette technique is approached for small droplets on the most slippery spikes (A) surface (red crosses), the relative error still remains more than 10 times lower when using MFS instead of Eq. (1). The legend in $\mathbf{g}$ also applies to the markers in $\mathbf{f}$ and $\mathbf{h}$. The error bars in all graphs are standard deviations or error propagations including these (see Supplementary Note 2).

underwater-SOCAL surface ${ }^{20}$ (Fig. 4). The etched silicon spikes surface thus represents a state-of-the-art, super-slippery superhydrophobic solid coating with the lowest measured dimensionless friction force to this date, challenging and even surpassing the slipperiness of next-generation coatings.

Rolling droplet dynamics. To investigate the internal fluid dynamics of the water droplets in our system, PIV experiments (Fig. 5a, b, Supplementary Movie 2 and Fig. 8a, b, Methods) were performed in conjunction with MFS on the spikes (A) and grass (E) samples at different substrate speeds $(v)$ and droplet radii $(R)$ (Supplementary Figs. 8c-d). Contrary to previous findings of water droplets sliding on superhydrophobic surfaces ${ }^{26,27}$, our droplets were, interestingly, found to roll with an angular velocity $\omega=v / R$ for low $v / R$ values (Fig. $5 c, d$ ), that is, like solid spheres without any slippage. In contrast to a sliplessly rotating solid sphere, however, our fluid droplets experience a velocity-independent, dissipative friction force also in the regime of slipless rolling (Supplementary Fig. 9). As $v / R$ was increased above a critical value $\left(\omega_{c}\right)$ in our experiments, the angular droplet velocity remained constant although the substrate speed was further increased or droplet size decreased (Fig. 5c, d). This can be understood through droplet-surface slippage ${ }^{46,47}$, with a slip speed $\left(v_{\mathrm{s}} / R=v / R-\omega\right)$ increasing as a function of $v / R$ (Supplementary Fig. 10). The transition to roll-slip motion occurred earlier on the spikes sample $\left(\omega_{\mathrm{c}}^{\mathrm{A}}=0.79 \pm 0.15 \mathrm{~s}^{-1}\right.$ vs. $\left.\omega_{\mathrm{c}}^{\mathrm{E}}=1.3 \pm 0.3 \mathrm{~s}^{-1}\right)$, which is reasonable since this has less liquidsolid interface (lower solid fraction, see Methods) to maintain a no-slip motion ${ }^{29}$.

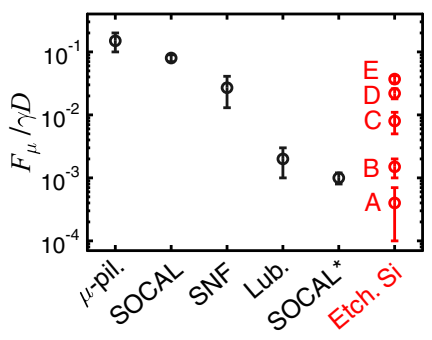

Fig. 4 Comparison between the friction on different liquid-repellent surfaces. The dimensionless friction force measured on: a micropillared ( $\mu$-pil.) surface with a solid fraction of 0.1 from ref. ${ }^{9}$, a SOCAL surface (in air) at drop speeds of $\sim 0.05 \mathrm{~mm} \mathrm{~s}^{-1}$ from ref. ${ }^{9}$, a silicone nanofilament (SNF) surface at drop speeds of $\sim 0.2 \mathrm{~mm} \mathrm{~s}^{-1}$ from ref. ${ }^{19}$, a lubricated surface at a capillary number of $\sim 10^{-5}$ from ref. ${ }^{9}$, a water-immersed polyzwitterionic brush (SOCAL*) surface with an oil droplet moving at a capillary number of $\sim 0^{-7}$ from Ref. ${ }^{20}$, and our etched silicon (Etch. Si) surfaces (samples A-E). The error bars for our etched silicon data (red markers) are error propagations including the standard deviations of $F_{\mu}$ and $D$ (see Supplementary Note 2). The data from our experiments on the etched silicon surfaces are averages from $n=56$ (A), 19 (B), 17 (C), 23 (D), and 17 (E) independent experiments performed repeatedly on the same samples.

The ratio between the translational $\left(E_{\mathrm{t}}=m v^{2} / 2\right.$, where $m$ is the droplet mass) and rotational kinetic energy $\left(E_{\mathrm{r}}=m R^{2} \omega^{2} / 5\right.$, assuming the moment of inertia of a solid sphere) of the droplet is $E_{\mathrm{t}} / E_{\mathrm{r}}=5(v / R)^{2} / 2 \omega^{2}$ (Fig. 5e). For $v / R<0.25 \pm 0.10 \mathrm{~s}^{-1}$, 

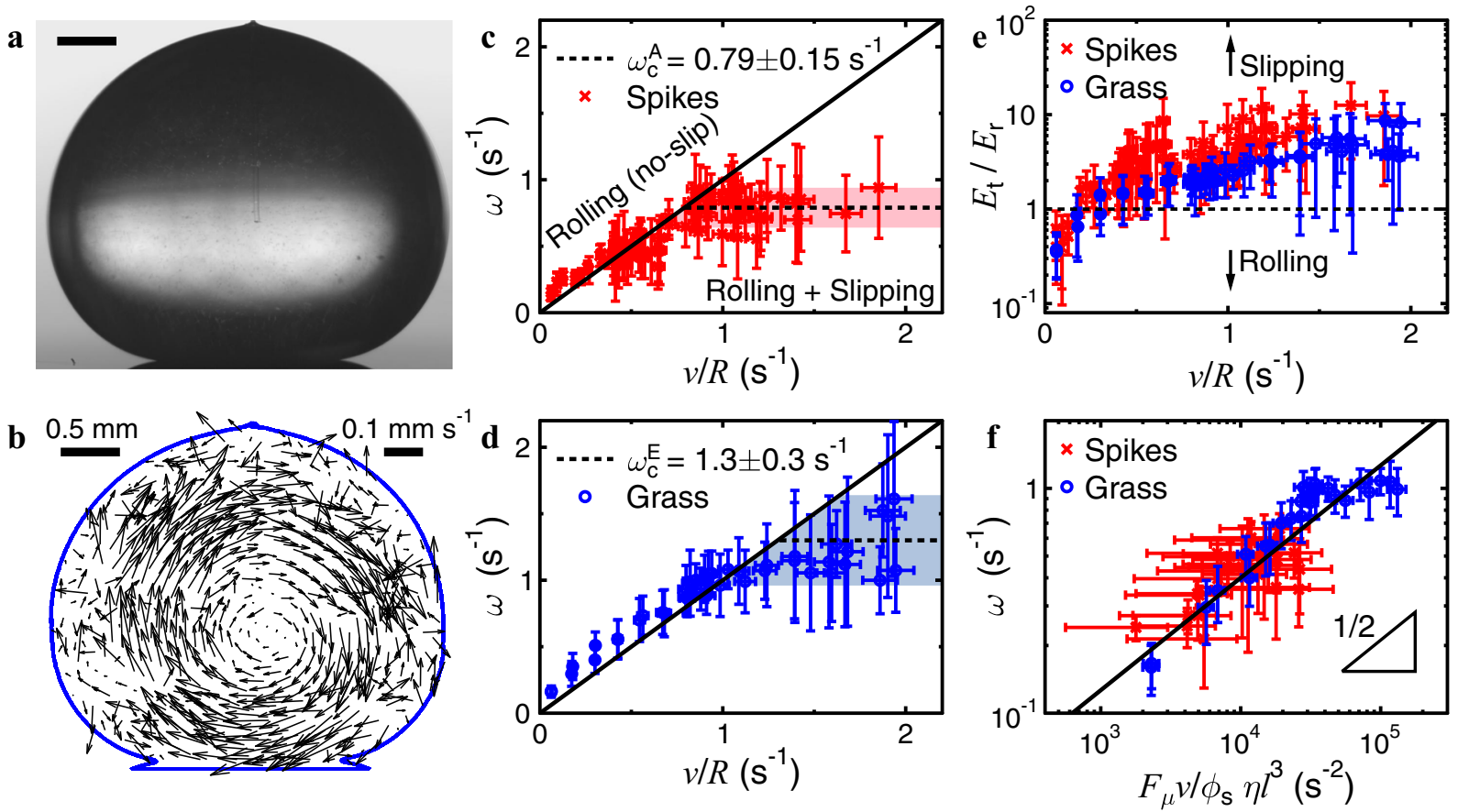

Fig. 5 Rolling vs. slipping droplets. a, b Particle image velocimetry experiments showing the rolling flow inside a water droplet $(R=1658 \pm 8 \mu \mathrm{m}$, substrate speed $v=0.1 \mathrm{~mm} \mathrm{~s}^{-1}$ to the left) on the spikes (A) surface. Scale bar in $\mathbf{a} 0.5 \mathrm{~mm}$. The blue line in $\mathbf{b}$ shows the outline of the droplet. $\mathbf{c}$, $\mathbf{d}$ Angular droplet velocity as a function of the ratio between sample speed and droplet radius $\left(v / R, v=0.1\right.$ to $1.9 \mathrm{~mm} \mathrm{~s}^{-1}$ and $R=0.8$ to $\left.1.6 \mathrm{~mm}\right)$ for the spikes (A) and grass (E) samples, respectively. Slowly rotating droplets behave as solid spheres rolling without any slippage ( $\omega=v / R$, solid line with a slope of unity). As $v / R$ exceeds a critical value ( $\omega_{\mathrm{c}}$, dashed line), $\omega$ remains constant and the rolling droplet starts slipping as $v / R$ is further increased. e The ratio between translational and rotational kinetic energy $\left(E_{\mathrm{t}} / E_{\mathrm{r}}=5(\mathrm{v} / R)^{2} / 2 \omega^{2}\right)$ as a function of $\mathrm{v} / R$ on the spikes $(\mathrm{A})$ and grass $(\mathrm{E})$ samples. $\mathbf{f}$ The angular velocity data for sliplessly rolling droplets $\left(v / R<\omega_{c}\right)$ on the spikes $(A)$ and grass $(E)$ samples collapse when plotted as a function of $F_{\mu} v / \eta \phi_{s} \beta$. The solid line in this $\log$ - log plot has a slope of $1 / 2$ as predicted by Eq. (2). The error bars in all graphs are standard deviations or error propagations including these (see Supplementary Note 2).

the rotational kinetic energy of our droplets is larger than their translational counterpart. It can be noted that the transition to roll-slip motion (based on $\omega_{\mathrm{c}}$ ) occurred at $E_{\mathrm{t}} / E_{\mathrm{r}} \approx 4$ on both surfaces. Previous to this study, the internal flow in water droplets on superhydrophobic surfaces has been studied only on tilted planes ${ }^{26,27}$, where $v \sim 10^{1} \mathrm{~cm} \mathrm{~s}^{-1}$ and $E_{\mathrm{t}}$ a factor of $\sim 10^{4}-10^{6}$ higher than in our slow experiments. For rotational speeds of $\omega<10^{2} \mathrm{~s}^{-1}$ (i.e., for all water droplets rolling without being strongly deformed by inertial effects), the motion on such tilted planes will remain in the translation-dominated, sliding regime. To the best of our knowledge, our MFS experiments thus allow for the first observation of purely rolling water droplets on superhydrophobic surfaces, as well as for a systematic investigation of the transition to roll-slip motion on these.

A droplet moving on a surface can experience viscous $\left(P_{\eta}\right)$, contact line $\left(P_{\mathrm{cl}}\right)$ and/or interfacial friction $\left(P_{\mathrm{f}}\right)$ energy dissipation ${ }^{47}$. In our system, $P_{\eta}$ dominates for purely rolling droplets $\left(v / R<\omega_{\mathrm{c}}\right)$, while $P_{\mathrm{cl}}$ and potentially also $P_{\mathrm{f}}$ become important in the roll-slip regime $\left(v / R>\omega_{c}\right.$, see Supplementary Note 4 for more details). For sliplessly rolling droplets, the rate of viscous dissipation in the Hertz volume near the liquid-solid interface is given by ${ }^{22,29,47} P_{\eta} \sim \eta \phi_{s} l^{3} \omega^{2}$, where $l=D / 2$ is the radius of the contact region, $\eta=0.001 \mathrm{~Pa}$ s is the viscosity of water, and $\phi_{\mathrm{s}}$ is the surface solid fraction (Methods). In our MFS PIV experiments (for $v / R<\omega_{\mathrm{c}}$ ), the viscous dissipation is balanced by $P_{\mathrm{d}} \sim F_{\mu} v$, so that $P_{\mathrm{d}} \sim P_{\eta}$. This gives a scaling prediction of the angular droplet velocity in the rolling regime

$$
\omega \sim\left(\frac{F_{\mu} v}{\eta \phi_{s} l^{3}}\right)^{1 / 2}
$$

In Fig. 5f, the experimental angular droplet velocity is plotted as a function of $F_{\mu} v / \eta \phi_{s} l^{3}$. The data collapses in accordance with Eq. (2).

To conclude, we study the friction of a surface that cannot be reliably measured with the gold standard method of contact angle goniometry, but instead needs to be characterized through direct force measurements. The dimensionless friction force of droplets moving on such liquid-repellent materials is suggested as the benchmark standard of the surface slipperiness. Our direct friction force measurements using micropipette force sensors render as much as three orders of magnitude more precise results as compared to using contact angle goniometry, and allow for the distinction between superhydrophobic samples with seemingly identical advancing and receding contact angles. A super-slippery superhydrophobic etched silicon surface is presented with a groundbreakingly low dimensionless friction force of $F_{\mu} / \gamma D=(4 \pm 3) \cdot 10^{-4}$, corresponding to a miniscule friction force of $7 \pm 4 \mathrm{nN}$ for a millimetric water droplet. This solid surface thus challenges and even surpasses the slipperiness of state-of-the-art, liquid-like coatings. Finally, we combine PIV with our micropipette force sensor measurements and reveal a previously unexplored droplet dynamics regime on superhydrophobic surfaces: slow water droplets are shown to roll without any 
slippage and transition to roll-slip motion as they start moving faster. The use of micropipette force sensors in droplet friction and dynamics experiments facilitates the search for even more slippery surfaces, and enables a comparison of surfaces with unprecedented sensitivity as the scientific community takes steps towards the extreme limit of slipperiness.

\section{Methods}

Micropipette force sensor measurements. We followed the MFS protocol ${ }^{42}$ when manufacturing and calibrating the micropipettes used in this work. In short, the micropipettes were pulled out of thick glass capillaries (i.d./o.d. $=0.75 / 1 \mathrm{~mm}$, World Precision Instruments, model no. TW100-6) using a micropipette puller (Narishige, model no. PN-31). The end of the micropipette was cut with a microforge (Narishige, model no. MF-900) to a cantilever length of $1.9-2.5 \mathrm{~cm}$, depending on the desired stiffness. The back end of the pipette was connected to a syringe and the micropipette was filled with MilliQ water. The micropipettes were then calibrated by mounting them horizontally and pushing out a small water droplet (density $\rho=1000 \mathrm{~kg} / \mathrm{m}^{3}$ ) to rest on the outside of the end of the pipette. The entire setup rested on an antivibration table (Halcyonics_i4large, Accurion). The experiment was recorded with a Phantom Miro M310 camera (with various resolutions, between $768 \times 768$ pix $^{2}$ and $1280 \times 800$ pix $^{2}$ ) at 24 fps using a macro lens (Canon MP-E $65 \mathrm{~mm} \mathrm{f/2.8} \mathrm{1-5x} \mathrm{Macro} \mathrm{Photo)} \mathrm{at} \mathrm{its} \mathrm{highest} \mathrm{magnification}$ $(\sim 3.99 \mu \mathrm{m} /$ pixel $)$. By varying the droplet size and analyzing its volume $(V)$ with a home-written Matlab code, the linear relation between the weight $(W=\rho V g)$ of the droplet and the micropipette deflection $(\Delta x$, analyzed with a home-written Matlab code) rendered the spring constant $\left(k_{\mathrm{p}}=W / \Delta x\right)$ of the cantilever. The calibration was repeated 3-8 times and the resulting average spring constant value was used together with its standard deviation. Many different micropipettes were manufactured and calibrated to be used for friction measurements on the different surfaces. The spring constants used in this work ranged between $k_{\mathrm{p}}=2.5-40 \mathrm{nN}$ $\mu \mathrm{m}^{-1}$ with relative errors $\sim 1-3 \%$ (Supplementary Table 3 ).

In a friction experiment, the water-filled, force-calibrated micropipette was mounted vertically above the sample resting on a motorized $x y z$-translational stage (Thorlabs, see photograph of the setup in Supplementary Fig. 1). A drop was pushed out from the micropipette tip until it slid down onto the surface, still attached to the micropipette tip. The stage was moved in the $z$-direction so that the micropipette end was centered inside the droplet (Fig. 2b). This was done merely to further ensure reproducibility between experiments. Since the micropipette diameter $(\sim 20$ $\mu \mathrm{m})$ is significantly smaller than the droplet diameter $(\sim 1 \mathrm{~mm})$, its location in the droplet is irrelevant to the internal fluid dynamics. Before each new measurement, the sample was moved in the $y$-direction (away from/towards the camera) to equilibrate the micropipette deflection in the $x$-direction (to the side as viewed from the camera) and place the droplet in a new position on the surface. In the experiment, the equilibrium, zero-force position of the micropipette was first recorded for $\sim 5 \mathrm{~s}$ and the substrate was then moved in the $x$-direction at a constant speed $\left(v=0.1 \mathrm{~mm} \mathrm{~s}^{-1}\right.$ with a relative error of $\left.5 \%\right)$ and acceleration $\left(a=4 \mathrm{~mm} \mathrm{~s}^{-2}\right)$ for $\sim 15-45 \mathrm{~s}$, depending on the surface. The experiment was recorded at a framerate of $50 \mathrm{fps}$ with the same camera and lens as used in the calibration. The kinetic friction force $\left(F_{\mu}\right)$ was determined as the difference between the average zero-force and kinetic friction regime (Fig. 2c). The micropipette deflection on the most slippery surfaces was barely visible by the naked eye, but could still be detected with the sub-pixel image analysis of the micropipette position ${ }^{42}$. The kinetic friction force was always analyzed in a regime of continuous droplet sliding, that is, where no pinning events (caused by defects in the coating) occurred. A static friction bump (Supplementary Fig. 11) was observed before the kinetic plateau in around $30 \%$ and $50 \%$ of the experiments on samples A and B, respectively, and in all experiments on samples C-E. The static jump likely occurred in every experiment also on the most slippery samples, but the force resolution of the MFS was not high enough to resolve all of these events. The static jump has been described in detail by others ${ }^{19}$, and this feature was not included in any further analysis in our work. The contact region diameter $(D)$ was measured using Matlab. See Supplementary Note 2 for details on the error analysis of all variables used in this work. The same sample was measured repeatedly to gain the data plotted in Figs. 3-4. A new spikes (A) sample was made for the measurements presented in Fig. 5, whereas the same grass (E) sample was used as in the pure friction force experiments.

Synthesis of etched silicon samples. The etched silicon samples were fabricated by maskless cryogenic deep reactive ion etching (Oxford Plasmalab System 100, Oxford Instruments, Bristol, UK) of silicon (the black silicon method) ${ }^{44}$. To obtain different topographies, $\mathrm{SF}_{6}$ gas flows (in $\mathrm{sccm}$ ) were $40,37.6,35.3,32.9$, and 30.5 , the $\mathrm{O}_{2}$ gas flows (in sccm) were 18,20.4, 22.8, 25.1, and 27.5, and the forward powers (in W) were $6,6,5,4$, and 4 for the spikes (A), B, C, D, and grass (E) samples, respectively. For all samples, the ICP power was $1000 \mathrm{~W}$, the etching temperature $-110^{\circ} \mathrm{C}$, the pressure $10 \mathrm{mTorr}$, and the etching time $7 \mathrm{~min}$. After etching, the samples were coated by a plasma-enhanced chemical vapor deposited (PECVD, Oxford Plasmalab 80+, Oxford Instruments, Bristol, UK) fluoropolymer. The parameters for depositing the coating were $50 \mathrm{~W}$ power, $250 \mathrm{mTorr}$ pressure, $100 \mathrm{sccm}$ of $\mathrm{CHF}_{3}$, and a deposition time of $5 \mathrm{~min}$.
Atomic force microscopy (AFM). The AFM measurements were carried out using Dimension Icon AFM (Bruker AXS, France; formerly Veeco) with ScanAsyst-air cantilever (sharp silicon tip with a nominal radius of $2 \mathrm{~nm}$ for PeakForce Tapping in air). Scan size was set to $10 \mu \mathrm{m} \times 10 \mu \mathrm{m}$ with 512 pix $\times 512$ pix resolution, and the scanning was done with a scan rate of $1 \mathrm{kHz}$. ScanAsyst Autocontrol was "on" for samples C-E with PeakForce Amplitude of $300 \mathrm{~nm}$, while was set to "Individual" for samples A-B with PeakForce Amplitude of $150 \mathrm{~nm}$. Spring constant and PeakForce frequency were $0.4 \mathrm{~N} \mathrm{~m}^{-1}$ and $2 \mathrm{kHz}$ for all samples. Individual scans for each sample were taken at five different locations on the surface. Representative AFM images of all samples are shown in Supplementary Fig. 4.

Scanning electron microscopy (SEM). The SEM imaging was carried out with a Zeiss Sigma VP scanning electron microscope. For the top-view imaging, samples were placed on carbon tape attached to an aluminum stub and coated with $5 \mathrm{~nm}$ gold-palladium coating using a Leica EM ACE600 high vacuum sputter coater before imaging. For the side-view imaging, samples were placed on a glass slide, which was vertically mounted in a sputter coater and coated with $8 \mathrm{~nm}$ goldpalladium coating before imaging. The imaging at $45^{\circ}$ tilt angle was done after sideview imaging by tilting the sample holder at $45^{\circ}$. The images were taken at low acceleration voltage of $1.0 \mathrm{kV}$ with an in-lens detector. Representative SEM images of all samples are shown in Supplementary Fig. 3.

Confocal microscopy. Confocal microscopy was carried out with Zeiss LSM710 confocal scanner attached to Zeiss Examiner upright microscope using $\times 20 / 1.0 \mathrm{~W}$ water immersion objective lens and $561 \mathrm{~nm}$ laser line. Imaging was done in reflection mode through a water droplet placed between the sample and the objective lens (Supplementary Fig. 5a), forming a plastron between the sample and the water droplet. The locations where the droplet is in direct contact with the substrate appear in the images as dark, whereas those locations with an air gap between the sample and the droplet appear as bright due to strong reflection from the water-air interface. A confocal image of the spikes (A) sample is shown in Supplementary Fig. 5b. The resolution of the confocal microscope was insufficient to image the contact zones on the other samples.

Contact angle goniometry (CAG). Conventional, optical contact angle measurements were performed using a contact angle goniometer (Biolin Attension Theta). Advancing and receding contact angles were measured separately (see Supplementary Method 1 for more details). The measurements were repeated 5-6 times at different positions on each surface.

Determination of the solid fraction. The solid fraction was difficult to quantify on the etched silicon samples due to the uneven shape of the pillars. Top-view SEM images (Supplementary Fig. 3o) were used to determine the solid fraction of the grass (E) sample, whereas top-view SEM (Supplementary Fig. 3k), AFM (Supplementary Fig. 4a) and confocal microscopy (Supplementary Fig. 5b) images were used to render an average solid fraction on the spikes (A) sample. Images were thresholded in Matlab and the fraction of the top parts of the posts was analyzed. An error of \pm 0.05 was assigned to the thresholding, rendering average solid fractions of $\phi_{\mathrm{s}}^{\mathrm{A}}=0.06 \pm 0.03$ and $\phi_{\mathrm{s}}^{\mathrm{E}}=0.47 \pm 0.05$.

Contact angle image analysis in the MFS experiments. A home-written Matlab code (Supplementary Code 1 video_CA.m) was used to analyze the front and back contact angles as a function of time in the MFS experiment. Each frame was made black and white, the outline of the droplet recognized and the thinnest part around the bottom of the drop defined as the contact line. The contact angles on both sides of the droplet were determined by fitting a fourth-degree polynomial to the data points along the boundary of the bottom half of the droplet, starting from the three-phase contact point (Supplementary Fig. 6a). The tangent of the polynomia was finally taken at the three-phase contact point. The average advancing and receding contact angles (Supplementary Fig. 6b) were calculated in the same time range as used to determine the kinetic friction force. The uncertainty in the contact angle analysis is described in detail in the Supplementary Method 1. This error was much higher than the standard deviation of the temporal average. The contact angles were reproduced with conventional contact angle goniometer measurements (see above and Supplementary Table 1).

Particle image velocimetry (PIV) experiments. A water droplet containing tracer particles $\left(5 \mu \mathrm{m}\right.$ polystyrene colloids with density $1.03 \mathrm{~g} \mathrm{ccm}^{-1}$; micromod Partikeltechnologie $\mathrm{GmbH}$, product code 30-19-503) was placed externally onto the micropipette and the surface. The PIV experiment was performed as in a normal friction force experiment (described above) using a framerate of $50 \mathrm{fps}$, a stiffer micropipette (length $1.7 \mathrm{~cm}, k_{\mathrm{p}}=40 \pm 0.8 \mathrm{nN} \mu \mathrm{m}^{-1}$ ) to keep the droplet more in place, and with the light source slightly shifted upwards to maximize the area of the drop where tracer particles could be seen. It should be noted that the edges of the droplet remained non-transparent and no data were used from the PIV analysis in this region. A range of substrate speeds $\left(v=0.1\right.$ to $\left.1.9 \mathrm{~mm} \mathrm{~s}^{-1}\right)$ and droplet sizes ( $R=0.8$ to $1.6 \mathrm{~mm}$ ) were used (Supplementary Fig. $8 \mathrm{c}-\mathrm{d}$ ) and the results were analyzed with Matlab's PIVLab code (see Supplementary Method 2 
for more details), rendering the average angular velocity $(\omega)$ of the internal rotational motion plotted in Fig. 5. The droplet shape remained unchanged during the experiments.

\section{Data availability}

The data presented in this paper āre available from the corresponding authors upon request.

Received: 19 May 2020; Accepted: 17 July 2020;

Published online: 15 September 2020

\section{References}

1. Holmberg, K. \& Erdemir, A. Influence of tribology on global energy consumption, costs and emissions. Friction 5, 263-284 (2017).

2. Simpson, J. T., Hunter, S. R. \& Aytug, T. Superhydrophobic materials and coatings: a review. Rep. Prog. Phys. 78, 086501 (2015).

3. Butt, H.-J. Characterization of super liquid-repellent surfaces. Curr. Opin. Colloid Interface Sci. 19, 343-354 (2014).

4. Kung, C. H., Sow, P. K., Zahiri, B. \& Mérida, W. Assessment and interpretation of surface wettability based on sessile droplet contact angle measurement: challenges and opportunities. Adv. Mater. Interfaces 6, 1900839 (2019).

5. Extrand, C. W. \& Kumagai, Y. Liquid drops on an inclined plane: the relation between contact angles, drop shape, and retentive force. J. Colloid Interface Sci. 170, 515-521 (1995).

6. ElSherbini, A. I. \& Jacobi, A. M. Retention forces and contact angles for critical liquid drops on non-horizontal surfaces. J. Colloid Interface Sci. 299, 841-849 (2006).

7. Pilat, D. W. et al. Dynamic measurement of the force required to move a liquid drop on a solid surface. Langmuir 28, 16812-16820 (2012).

8. Daniel, D., Timonen, J. V. I., Li, R., Velling, S. J. \& Aizenberg, J. Oleoplaning droplets on lubricated surfaces. Nat. Phys. 13, 1020-1025 (2017).

9. Daniel, D. et al. Origins of liquid-repellency on structured, flat, and lubricated surfaces. Phys. Rev. Lett. 120, 244503 (2018).

10. Wooh, S. \& Vollmer, D. Silicone brushes: omniphobic surfaces with low sliding angles. Angew. Chem. Int. Ed. 55, 6822-6824 (2016).

11. Quéré, D. Wetting and roughness. Annu. Rev. Mater. Res. 38, 71-99 (2008).

12. Srinivasan, S., McKinley, G. H. \& Cohen, R. E. Assessing the accuracy of contact angle measurements for sessile drops on liquid-repellent surfaces. Langmuir 27, 13582-13589 (2011).

13. Liu, K., Vuckovac, M., Latikka, M., Huhtamäki, T. \& Ras, R. H. A. Improving surface-wetting characterization. Science 363, 1147-1148 (2019).

14. Vuckovac, M., Latikka, M., Liu, K., Huhtamäki, T. \& Ras, R. H. A. Uncertainties in contact angle goniometry. Soft Matter 15, 7089-7096 (2019).

15. Pierce, E., Carmona, F. J. \& Amirfazli, A. Understanding of sliding and contact angle results in tilted plate experiments. Colloids Surf. Physicochem. Eng. Asp. 323, 73-82 (2008).

16. Mouterde, T., Raux, P. S., Clanet, C. \& Quéré, D. Superhydrophobic frictions. Proc. Natl. Acad. Sci. USA 116, 8220-8223 (2019).

17. Timonen, J. V. I., Latikka, M., Ikkala, O. \& Ras, R. H. A. Free-decay and resonant methods for investigating the fundamental limit of superhydrophobicity. Nat. Commun. 4, 2398 (2013).

18. Suda, H. \& Yamada, S. Force measurements for the movement of a water drop on a surface with a surface tension gradient. Langmuir 19, 529-531 (2003).

19. Gao, N. et al. How drops start sliding over solid surfaces. Nat. Phys. 14, 191-196 (2018).

20. Daniel, D. et al. Hydration lubrication of polyzwitterionic brushes leads to nearly friction- and adhesion-free droplet motion. Commun. Phys. 2, 105 (2019).

21. Mognetti, B. M., Kusumaatmaja, H. \& Yeomans, J. M. Drop dynamics on hydrophobic and superhydrophobic surfaces. Faraday Discuss. 146, 153-165 (2010).

22. Mahadevan, L. \& Pomeau, Y. Rolling droplets. Phys. Fluids 11, 2449-2453 (1999).

23. Richard, D. \& Quéré, D. Viscous drops rolling on a tilted non-wettable solid. Europhys. Lett. 48, 286-291 (1999).

24. Suzuki, S. et al. Slipping and rolling ratio of sliding acceleration for a water droplet sliding on fluoroalkylsilane coatings of different roughness. Chem. Lett. 37, 58-59 (2008).

25. Sakai, M. et al. Image analysis system for evaluating sliding behavior of a liquid droplet on a hydrophobic surface. Rev. Sci. Instrum. 78, 045103 (2007).

26. Sakai, M. et al. Direct observation of internal fluidity in a water droplet during sliding on hydrophobic surfaces. Langmuir 22, 4906-4909 (2006).
27. Sakai, M. et al. Sliding of water droplets on the superhydrophobic surface with $\mathrm{ZnO}$ nanorods. Langmuir 25, 14182-14186 (2009).

28. Choi, C.-H. \& Kim, C.-J. Large slip of aqueous liquid flow over a nanoengineered superhydrophobic surface. Phys. Rev. Lett. 96, 066001 (2006).

29. Ybert, C., Barentin, C., Cottin-Bizonne, C., Joseph, P. \& Bocquet, L. Achieving large slip with superhydrophobic surfaces: Scaling laws for generic geometries. Phys. Fluids 19, 123601 (2007).

30. Colbert, M.-J., Raegen, A. N., Fradin, C. \& Dalnoki-Veress, K. Adhesion and membrane tension of single vesicles and living cells using a micropipettebased technique. Eur. Phys. J. E 30, 117 (2009).

31. Colbert, M.-J., Brochard-Wyart, F., Fradin, C. \& Dalnoki-Veress, K. Squeezing and detachment of living cells. Biophys. J. 99, 3555-3562 (2010).

32. Backholm, M., Ryu, W. S. \& Dalnoki-Veress, K. Viscoelastic properties of the nematode Caenorhabditis elegans, a self-similar, shear-thinning worm. Proc. Natl. Acad. Sci. USA 110, 4528-4533 (2013).

33. Schulman, R. D., Backholm, M., Ryu, W. S. \& Dalnoki-Veress, K. Dynamic force patterns of an undulatory microswimmer. Phys. Rev. E 89, 050701 (2014)

34. Schulman, R. D., Backholm, M., Ryu, W. S. \& Dalnoki-Veress, K. Undulatory microswimming near solid boundaries. Phys. Fluids 26, 101902 (2014).

35. Backholm, M., Kasper, A. K. S., Schulman, R. D., Ryu, W. S. \& DalnokiVeress, $\mathrm{K}$. The effects of viscosity on the undulatory swimming dynamics of C. elegans. Phys. Fluids 27, 091901 (2015).

36. Backholm, M., Ryu, W. S. \& Dalnoki-Veress, K. The nematode C. elegans as a complex viscoelastic fluid. Eur. Phys. J. E 38, 36 (2015).

37. Petit, J. et al. A modular approach for multifunctional polymersomes with controlled adhesive properties. Soft Matter 14, 894-900 (2018).

38. Kreis, C. T., Le Blay, M., Linne, C., Makowski, M. M. \& Bäumchen, O. Adhesion of Chlamydomonas microalgae to surfaces is switchable by light. Nat. Phys. 14, 45-49 (2018).

39. Böddeker, T. J., Karpitschka, S., Kreis, C. T., Magdelaine, Q. \& Bäumchen, O. Dynamic force measurements on swimming Chlamydomonas cells using micropipette force sensors. J. R. Soc. Interface 17, 20190580 (2020).

40. Ono-dit-Biot, J.-C. et al. Rearrangement of two dimensional aggregates of droplets under compression: Signatures of the energy landscape from crystal to glass. Phys. Rev. Res. 2, 023070 (2020).

41. Ono-Dit-Biot, J.-C. et al. Mechanical properties of model colloidal monocrystals. arXiv http://arxiv.org/abs/2007.00917 (2020).

42. Backholm, M. \& Bäumchen, O. Micropipette force sensors for in vivo force measurements on single cells and multicellular microorganisms. Nat. Protoc 14, 594-615 (2019).

43. Francis, G. W., Fisher, L. R., Gamble, R. A. \& Gingell, D. Direct measurement of cell detachment force on single cells using a new electromechanical method. J. Cell Sci. 87, 519-523 (1987).

44. Sainiemi, L. et al. Non-reflecting silicon and polymer surfaces by plasma etching and replication. Adv. Mater. 23, 122-126 (2011).

45. Qiao, S. et al. Friction of droplets sliding on microstructured superhydrophobic surfaces. Langmuir 33, 13480-13489 (2017).

46. Rothstein, J. P. Slip on Superhydrophobic Surfaces. Annu. Rev. Fluid Mech. 42 89-109 (2010).

47. Smith, A. F. W., Mahelona, K. \& Hendy, S. C. Rolling and slipping of droplets on superhydrophobic surfaces. Phys. Rev. E 98, 033113 (2018).

\section{Acknowledgements}

This work was supported by the Academy of Finland (Centres of Excellence Programme (2014-2019), Postdoctoral Research Grant (grant agreement number 309237), and Academy of Finland Project (grant agreement number 297360)), as well as the Aalto University AScI International Internship Programme and AScI/ELEC Thematic Research Programme. R.H.A.R. acknowledges the European Research Council for funding the Consolidator Grant SuperRepel (grant agreement number 725513). We acknowledge the provision of facilities by Aalto University at OtaNano-Micronova Nanofabrication Centre and Nanomicroscopy Center (Aalto-NMC).

\section{Author contributions}

R.H.A.R. and M.B. conceived the idea of the experiments. M.B. performed the friction force measurements together with D.M. The PIV experiments were performed and analyzed by D.M. under the supervision of M.B. M.V. performed the AFM and SEM measurements as well as the contact angle error analysis. M.J.H. performed the conventional contact angle goniometry measurements, and H.N. wrote the time-resolved contact angle analysis code used in the MFS analysis. V.J. made all etched silicon samples and J.V.I.T. performed the confocal microscopy measurements. M.B. analyzed the final data and developed the theory. M.B. discussed the results with all authors and wrote the manuscript with input from all coauthors. 


\section{Competing interests}

M.V., J.V.I.T., and R.H.A.R. are inventors of patent applications on wetting characterization techniques, and are together with M.J.H. considering potential commercialization partly supported by Business Finland and ERC. M.B., D.M., H.N., and V.J. declare no competing interests.

\section{Additional information}

Supplementary information is available for this paper at https://doi.org/10.1038/s43246020-00065-3.

Correspondence and requests for materials should be addressed to M.B. or R.H.A.R.

Reprints and permission information is available at http://www.nature.com/reprints

Publisher's note Springer Nature remains neutral with regard to jurisdictional claims in published maps and institutional affiliations. (c) (i) Open Access This article is licensed under a Creative Commons Attribution 4.0 International License, which permits use, sharing, adaptation, distribution and reproduction in any medium or format, as long as you give appropriate credit to the original author(s) and the source, provide a link to the Creative Commons license, and indicate if changes were made. The images or other third party material in this article are included in the article's Creative Commons license, unless indicated otherwise in a credit line to the material. If material is not included in the article's Creative Commons license and your intended use is not permitted by statutory regulation or exceeds the permitted use, you will need to obtain permission directly from the copyright holder. To view a copy of this license, visit http://creativecommons.org/ licenses/by/4.0/.

(C) The Author(s) 2020 